\title{
Application of Generalized Integral Method (GIRM) to Numerical Evaluations of Soliton-to-Soliton and Soliton-to-Bottom Interactions
}

\author{
Gantulga Tsedendorj ${ }^{1}$, Hiroshi Isshiki ${ }^{2}$, Rinchinbazar Ravsal ${ }^{3}$ \\ ${ }^{1}$ Department of Mathematics, National University of Mongolia, Ulaanbaatar, Mongolia \\ ${ }^{2}$ IMA, Institute of Mathematical Analysis, Osaka, Japan \\ ${ }^{3}$ Department of Administration, National University of Mongolia, Ulaanbaatar, Mongolia
}

Email address:

gantulga@smcs.num.edu.mn (G. Tsedendorj), isshiki@dab.hi-ho.ne.jp (H. Isshiki), rinchinbazar_r@num.edu.mn (R. Ravsal)

\section{To cite this article:}

Gantulga Tsedendorj, Hiroshi Isshiki, Rinchinbazar Ravsal. Application of Generalized Integral Method (GIRM) to Numerical Evaluations of Soliton-to-Soliton and Soliton-to-Bottom Interactions. Applied and Computational Mathematics. Special Issue: Integral Representation Method and its Generalization. Vol. 4, No. 3-1, 2015, pp. 78-86. doi: 10.11648/j.acm.s.2015040301.16

\begin{abstract}
Numerical evaluations of soliton-soliton and soliton-to-bottom interaction have many applications in various fields. On the other hand, Generalized Integral Representation Method (GIRM) is known as a convenient numerical method for solving Initial and Boundary Value Problem of differential equations such as advective diffusion. In this work, we apply one-step GIRM to numerical evaluations of propagation of a single soliton, soliton-to-soliton interaction and soliton-to-bottom interaction. Firstly, in case of a single soliton, the bottom is considered to be constant in order to understand the behavior of the soliton propagation as it travels in the middle of the sea. Next, in case of soliton-to-bottom, we study behavior of a single soliton propagation when the bottom has different geometries. Finally, we evaluate interaction of two different i.e., big and small solitons. To carry out with the studies, we derive and implement GIRM to numerically solve the Korteweg-de Vries (KdV) equation. In order to verify the theory, numerical experiments are conducted and accurate approximate solutions are obtained in each case of the soliton interactions.
\end{abstract}

Keywords: Korteweg-de Vries (KdV) equation, Single Soliton, Soliton-to-Soliton interaction, Soliton-to-Bottom interaction, Numerical Evaluation, Generalized Integral Representation Method (GIRM)

\section{Introduction}

The propagation of solitary waves has many applications in various fields in particular, in tsunami studies. Solitary waves or solitons are propagating through a certain medium with constant velocity and describe a variety of nonlinear wave phenomena in one dimension. This phenomenon was first observed, described and reproduced by J.S. Russell [1].

It is well-known that the nonlinear wave equation of Korteweg and de Vries (KdV) [2] with the appropriate initial conditions admits solitons as its solutions. A modified version of the $\mathrm{KdV}$ equation with the variable-depth is studied in Ref [3]. Some derivations of the variable-depth $\mathrm{KdV}$ equation that allow a bottom profile are discussed in Ref [3] and [5]. Another approach, spectral and pseudo-spectral numerical schemes for the KdV equation are discussed in Ref. [4].

In this study, we develop and implement a high precision numerical scheme based on GIRM, in order to study and evaluate a single soliton propagation, soliton-to-bottom and soliton-to-soliton interactions. GIRM is known as a convenient alternative method for solving Initial and Boundary Value Problem of differential equations such as advective diffusion. In Ref. [6], GIRM is applied to one- and two-dimensional diffusion problems and two-dimensional Burgers' equation. It is also shown that the determination of a fundamental solution for GIRM is always possible in advance. Effects of Generalized Fundamental Solution (GFS) on GIRM is discussed in Ref. [7]. Further, GIRM is applied to fluid dynamic motion of gas in Ref. [8] and to tidal wave propagation in Ref [9] in order to obtain the accurate numerical solutions. Full computer codes of One and Two-step GIRMs written in widely used computational languages such as Matlab, C, and FORTRAN are given in Ref [10]. 


\section{Theory of 1-Step GIRM}

\subsection{KdV Equation}

Let $x$ and $t$ be the coordinate and time $\eta(x, t)$ is the surface elevation. The Kortweg-de Vries or KdV equation is given by

$$
\frac{\partial \eta}{\partial t}+c_{0} \frac{\partial \eta}{\partial x}+k_{N} \eta \frac{\partial \eta}{\partial x}+k_{D} \frac{\partial^{3} \eta}{\partial x^{3}}=0
$$

with

$$
k_{N}=\frac{3 c_{0}}{2 h} \text { and } k_{D}=\frac{c_{0} h^{2}}{6}
$$

where $h$ and $c_{0}$ are the depth of water and speed of the linear wave, respectively. KdV equation was obtained under the assumption that the water depth $h$ is constant. However, if the water depth changes slowly, $\mathrm{KdV}$ equation could reflect this change. Hence, we consider $h$ and $c_{0}$ as functions of $x$ and $t$ i.e. $h=h(x, t)$ and $c_{0}=c_{0}(x, t)$. The linear wave speed $c_{0}(x, t)$ is given by

$$
c_{0}(x, t)=\sqrt{g h(x, t)}
$$

with $g$ is the gravitational acceleration. If we rewrite Eq. (1) in a more general form, we have

$$
\begin{aligned}
& \frac{\partial \eta(x, t)}{\partial t}+c_{0}(x, t) \frac{\partial \eta(x, t)}{\partial x}+\frac{1}{2} k_{N}(x, t) \frac{\partial \eta^{2}(x, t)}{\partial x} \\
& +k_{D}(x, t) \frac{\partial^{3} \eta(x, t)}{\partial x^{3}}+\kappa(x, t) \frac{\partial^{2} \eta(x, t)}{\partial x^{2}}=0
\end{aligned}
$$

In Eq. (4), diffusion term $\kappa(x, t) \partial^{2} \eta(x, t) / \partial x^{2}$ is added, where $\kappa$ is the diffusion coefficient. The wave form and the speed of a soliton obtained analytically are given by

$$
\begin{aligned}
& \eta=\eta_{0} \operatorname{sech}^{2}\left(\sqrt{\frac{3 \eta_{0}}{4 h^{3}}}(x-c t)\right) \\
& c=c_{0}\left(1+\frac{\eta_{0}}{2 h}\right)=\sqrt{g h}\left(1+\frac{\eta_{0}}{2 h}\right) .
\end{aligned}
$$

\subsection{One-Step GIRM for KdV Equation}

First, we derive 1-step Generalized Integral Representation (GIR) for Eq. (4). Since KdV equation expresses a wave traveling to the positive direction of $x$, we consider it in the region $0<x<L$, where $L$ is large enough. We also assume function $\eta$ and its derivatives tend to zero, as $x$ tends to 0 and $L$.

Multiplying both sides of Eq. (4) by function $\tilde{G}(x, \xi)$ of $x$ and $\xi$, and integrating over $0<x<L$, we obtain

$$
\begin{aligned}
& 0=\int_{0}^{L} \tilde{G}(x, \xi)\left[\frac{\partial \eta(x, t)}{\partial t}+c_{0}(x, t) \frac{\partial \eta(x, t)}{\partial x}+\frac{1}{2} k_{N}(x, t) \frac{\partial \eta^{2}(x, t)}{\partial x}\right. \\
& \left.+k_{D}(x, t) \frac{\partial^{3} \eta(x, t)}{\partial x^{3}}+\kappa(x, t) \frac{\partial^{2} \eta(x, t)}{\partial x^{2}}\right] d x
\end{aligned}
$$

Next, transforming all the spatial derivatives of $\eta$ in Eq. (7) and taking into account the boundary conditions, yield

$$
\begin{aligned}
& 0=\int_{0}^{L} \tilde{G}(x, \xi) \frac{\partial \eta(x, t)}{\partial t} d x \\
& -\int_{0}^{L} \eta(x, t) \frac{\partial}{\partial x}\left(c_{0}(x, t) \tilde{G}(x, \xi)\right) d x \\
& -\frac{1}{2} \int_{0}^{L} \eta^{2}(x, t) \frac{\partial}{\partial x}\left(k_{N}(x, t) \tilde{G}(x, \xi)\right) d x \\
& -\int_{0}^{L} \eta(x, t) \frac{\partial^{3}}{\partial x^{3}}\left(k_{D}(x, t) \tilde{G}(x, \xi)\right) d x \\
& +\int_{0}^{L} \eta(x, t) \frac{\partial^{2}}{\partial x^{2}}(\kappa(x, t) \tilde{G}(x, \xi)) d x
\end{aligned}
$$

Finally, rearranging and exchanging variables $x$ and $\xi$, in Eq. (8), we have

$$
\begin{aligned}
& \int_{0}^{L} \frac{\partial \eta(\xi, t)}{\partial t} \tilde{G}(\xi, x) d \xi= \\
& {\left[\begin{array}{l}
\frac{\partial}{\partial \xi}\left(c_{0}(\xi, t) \tilde{G}(\xi, x)\right) \eta(\xi, t)+ \\
\frac{1}{2} \frac{\partial}{\partial \xi}\left(k_{N}(\xi, t) \tilde{G}(\xi, x)\right) \eta^{2}(\xi, t)+ \\
\int_{0}^{L}\left[\begin{array}{l}
\frac{\partial^{3}}{\partial \xi^{3}}\left(k_{D}(\xi, t) \tilde{G}(\xi, x)\right) \eta(\xi, t)- \\
\frac{\partial^{2}}{\partial \xi^{2}}(\kappa(\xi, t) \tilde{G}(\xi, x)) \eta(\xi, t)
\end{array}\right] d \xi
\end{array}\right.}
\end{aligned}
$$

where $\tilde{G}(x, \xi)$ is a Generalized Fundamental Solution (GFS) chosen properly. The determination of a fundamental solution for GIRM is always possible in advance [6-10]. In our case, we take the Gaussian GFS:

$$
\tilde{G}(x, \xi)=\frac{1}{\sqrt{2 \pi} \gamma} \exp \left(-\frac{(x-\xi)^{2}}{2 \gamma^{2}}\right)
$$

Eq. (9) is a GIR of Eq. (4). This integral representation is applied to numerical solution of Eq. (4). If $\eta(x, t)$ is known in $0 \leq x \leq L$, then Eq. (9) is an integral equation with unknowns $\partial \eta(x, t) / \partial t$ in $0<x<L$, where $\tilde{G}(x, \xi)$ is the kernel function of the integral equation. Namely, we can obtain $\eta(x, t)$ numerically, if we use, for instance, the following procedure:

Let $\eta(x, t)$ be known at time $t \rightarrow$

Obtain $\partial \eta(x, t) / \partial t$ from Eq. (9) $\rightarrow$

Then $\eta(x, t+d t)=\eta(x, t)+d t \partial \eta(x, t) / \partial t \rightarrow$

Add $d t$ to $t \rightarrow$ Repeat process.

In the present paper, an iteration is used to obtain $\partial \eta(x, t+d t) / \partial t \quad(\rightarrow$ Appendix A $)$. 


\section{Numerical Method and Results of 1-Step GIRM}
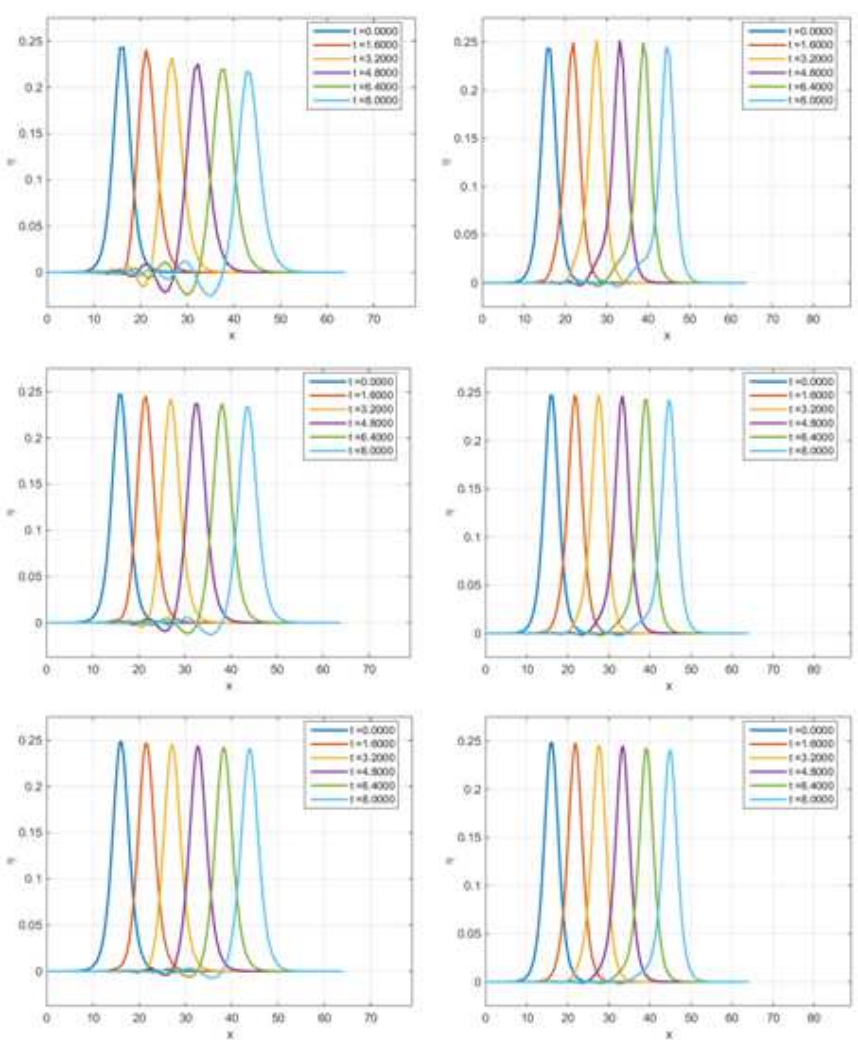

(a)

(b)

Figure 1. Numerical solutions of propagation of a single soliton (a) by Runge-Kutta method, (b) by 1-step GIRM, where N=80, 120, 160 (from top to bottom).

In numerical experiments, we take for simplicity, the coefficients $c_{0}(x, t), k_{N}(x, t), k_{D}(x, t)$ and $\kappa(x, t)$ to change slowly with respect to $x$. In this case, Eq. (9) would be approximated by

$$
\begin{aligned}
& \int_{0}^{L} \frac{\partial \eta(\xi, t)}{\partial t} \tilde{G}(\xi, x) d \xi= \\
& \int_{0}^{L} \eta(\xi, t) c_{0}(\xi, t) \frac{\partial \tilde{G}(\xi, x)}{\partial \xi} d \xi+ \\
& \frac{1}{2} \int_{0}^{L} \eta^{2}(\xi, t) k_{N}(\xi, t) \frac{\partial \tilde{G}(\xi, x)}{\partial \xi} d \xi+ \\
& \int_{0}^{L} \eta(\xi, t) k_{D}(\xi, t) \frac{\partial^{3} \tilde{G}(\xi, x)}{\partial \xi^{3}} d \xi- \\
& \int_{0}^{L} \eta(\xi, t) \kappa(\xi, t) \frac{\partial^{2} \tilde{G}(\xi, x)}{\partial \xi^{2}} d \xi
\end{aligned}
$$

To begin with, we introduce a uniform mesh as follows:

$$
d x=d \xi=L / N, x_{i}=\xi_{i}=(i+0.5) d x, \begin{gathered}
i=0,1, \ldots, N-1, \\
n=0,1, \ldots
\end{gathered} t_{n}=n d t,
$$

and

$$
\begin{gathered}
\eta_{i}^{(n)}=\eta\left(x_{i}, t_{n}\right),\left[\frac{\partial \eta}{\partial t}\right]_{j}^{(n)}=\frac{\partial \eta\left(\xi_{j}, t_{n}\right)}{\partial t}, c_{0 i}^{(n)}=c_{0}\left(x_{i}, t_{n}\right), \\
k_{N i}^{(n)}=k_{N}\left(x_{i}, t_{n}\right) \text { etc. }
\end{gathered}
$$

We approximate each term in Eq. (12) as follows:

$$
\begin{aligned}
& \int_{0}^{L} \frac{\partial \eta\left(\xi, t_{n}\right)}{\partial t} \tilde{G}(x, \xi) d \xi= \\
& \sum_{j=0}^{N-1} \int_{\xi_{j}-d \xi / 2}^{\xi_{j}+d \xi / 2} \frac{\partial \eta\left(\xi, t_{n}\right)}{\partial t} \tilde{G}(x, \xi) d \xi= \\
& \sum_{j=0}^{N-1}\left[\frac{\partial \eta}{\partial t}\right]_{j}^{(n)} \int_{\xi_{j}-d \xi / 2}^{\xi_{j}+d \xi / 2} \tilde{G}(x, \xi) d \xi \\
& \int_{0}^{L} \eta\left(\xi, t_{n}\right) c_{0}\left(\xi, t_{n}\right) \frac{\partial \tilde{G}(\xi, x)}{\partial \xi} d \xi= \\
& \sum_{j=0}^{N-1} \int_{\xi_{j}-d \xi / 2}^{\xi_{j}+d \xi / 2} \eta\left(\xi, t_{n}\right) c_{0}\left(\xi, t_{n}\right) \frac{\partial \tilde{G}(x, \xi)}{\partial \xi} d \xi= \\
& \sum_{j=0}^{N-1} \eta_{j}^{(n)} c_{0 j}^{(n)} \int_{\xi_{j}-d \xi / 2}^{\xi_{j}+d \xi / 2} \frac{\partial \tilde{G}(x, \xi)}{\partial \xi} d \xi \\
& \int_{0}^{L} \eta^{2}\left(\xi, t_{n}\right) k_{N}\left(\xi, t_{n}\right) \frac{\partial \tilde{G}(\xi, x)}{\partial \xi} d \xi= \\
& \sum_{j=0}^{N-1} \int_{\xi_{j}-d \xi / 2}^{\xi_{j}+d \xi / 2} \eta^{2}\left(\xi, t_{n}\right) k_{N}\left(\xi, t_{n}\right) \frac{\partial \tilde{G}(x, \xi)}{\partial \xi} d \xi= \\
& \sum_{j=0}^{N-1}\left(\eta_{j}^{(n)}\right)^{2} k_{N j}^{(n)} \int_{\xi_{j}-d \xi / 2}^{\xi_{j}+d \xi / 2} \frac{\partial \tilde{G}(x, \xi)}{\partial \xi} d \xi \\
& \int_{0}^{L} \eta\left(\xi, t_{n}\right) k_{D}\left(\xi, t_{n}\right) \frac{\partial^{3} \tilde{G}(\xi, x)}{\partial \xi^{3}} d \xi= \\
& \sum_{j=0}^{N-1} \int_{\xi_{j}-d \xi / 2}^{\xi_{j}+d \xi / 2} \eta\left(\xi, t_{n}\right) k_{D}\left(\xi, t_{n}\right) \frac{\partial^{3} \tilde{G}(x, \xi)}{\partial \xi^{3}} d \xi= \\
& \sum_{j=0}^{N-1} \eta_{j}^{(n)} k_{D j}^{(n)} \int_{\xi_{j}-d \xi / 2}^{\xi_{j}+d \xi / 2} \frac{\partial^{3} \tilde{G}(x, \xi)}{\partial \xi^{3}} d \xi
\end{aligned}
$$

Hence Eq. (12) can be discretized as

$$
\begin{aligned}
& \sum_{j=0}^{N-1}\left[\frac{\partial \eta}{\partial t}\right]_{j}^{(n)} \Gamma_{j}(x)=\sum_{j=0}^{N-1} \eta_{j}^{(n)} c_{0 j}^{(n)} \Lambda_{j}(x)+ \\
& \frac{1}{2} \sum_{j=0}^{N-1}\left(\eta_{j}^{(n)}\right)^{2} k_{N j}^{(n)} \Lambda_{j}(x)-\kappa \varepsilon(x) \eta(x, t)+\sum_{j=0}^{N-1} \eta_{j}^{(n)} k_{D j}^{(n)} \Theta_{j}(x)
\end{aligned}
$$

where we denote

$$
\begin{gathered}
\Gamma_{j}(x)=\int_{\xi_{j}-d \xi / 2}^{\xi_{j}+d \xi / 2} \tilde{G}(x, \xi) d \xi \\
\Lambda_{j}(x)=\int_{\xi_{j}-d \xi / 2}^{\xi_{j}+d \xi / 2} \frac{\partial \tilde{G}(x, \xi)}{\partial \xi} d \xi \\
\Theta_{j}(x)=\int_{\xi_{j}-d \xi / 2}^{\xi_{j}+d \xi / 2} \frac{\partial^{3} \tilde{G}(x, \xi)}{\partial \xi^{3}} d \xi
\end{gathered}
$$

and 


$$
\varepsilon(x)=\left\{\begin{array}{l}
1, \text { if } 0<x<L \\
0.5, \text { if } x=0 \text { or } x=L \\
0, \text { otherwise }
\end{array}\right.
$$

Eq. (16) is satisfied at the points $x=x_{0}, x_{1}, \cdots, x_{N-1}$ and the unknowns are $[\partial \eta / \partial t]_{j}^{(n)}, j=0,1, \ldots, N-1$. Hence, we have $\mathrm{N}$ equations for $\mathrm{N}$ unknowns.

\subsection{Propagation of a Single Soliton}

First, we numerically evaluate propagation of a single soliton as if it moves in an infinite open sea. Numerical experiment is conducted straightforward by using Eq. (16) along with Eq. (17a) and Eq. (17b). For comparison, we also evaluate it by Finite Difference Method using second order Runge-Kutta scheme. Numerical results are shown in Fig. 1. The accuracy of the numerical results by 1-step GIRM using implicit time evolution is high. Values of the parameters used in the numerical experiments are:

$g=9.8, \eta_{0}=0.25, h=1, L=64, N=80,120,160, d x=L / N, d t=0.004$,

$$
T=2000 d t, \quad \gamma=0.75 d x
$$

and the initial condition is

$$
\eta(x, 0)=\eta_{0} \operatorname{sech}^{2}\left(\sqrt{\frac{3 \eta_{0}}{4 h^{3}}}\left(x-\frac{L}{4}\right)\right)
$$

We provide Matlab code for propagation of a single soliton in Appendix A.

\subsection{Soliton-to-Soliton Interaction}

We consider an interaction of big and small solitons. From Eq. (6), the big one moves faster than the smaller one. Hence, the big one catches up the small one. We introduce a frame moving with mean speed of the individual solitons:

$$
\begin{gathered}
x^{\prime}=x-U t, t^{\prime}=t \\
\frac{\partial}{\partial x}=\frac{\partial x^{\prime}}{\partial x} \frac{\partial}{\partial x^{\prime}}+\frac{\partial t^{\prime}}{\partial x} \frac{\partial}{\partial t^{\prime}}=\frac{\partial}{\partial x^{\prime}} \\
\frac{\partial}{\partial t}=\frac{\partial x^{\prime}}{\partial t} \frac{\partial}{\partial x^{\prime}}+\frac{\partial t^{\prime}}{\partial t} \frac{\partial}{\partial t^{\prime}}=-U \frac{\partial}{\partial x^{\prime}}+\frac{\partial}{\partial t^{\prime}}
\end{gathered}
$$

$\frac{\partial}{\partial x^{2}}=\frac{\partial}{\partial x^{\prime 2}}$ and $\frac{\partial}{\partial x^{3}}=\frac{\partial}{\partial x^{\prime 3}}$, then Eq. (1) becomes

$$
\frac{\partial \eta}{\partial t^{\prime}}+\left(c_{0}-U\right) \frac{\partial \eta}{\partial x^{\prime}}+k_{N} \eta \frac{\partial \eta}{\partial x^{\prime}}+k_{D} \frac{\partial^{3} \eta}{\partial x^{\prime 3}}=0
$$

Therefore, a GIR of Eq. (20) is

$$
\left.\begin{array}{l}
\int_{0}^{L} \frac{\partial \eta(\xi, t)}{\partial t} \tilde{\eta}(\xi, x, t) d \xi= \\
\int_{0}^{L}\left[\begin{array}{l}
\frac{\partial\left(c_{0}(\xi, t)-U\right) \tilde{\eta}(\xi, x)}{\partial \xi} \eta(\xi, t)+ \\
\frac{1}{2} \frac{\partial k_{N}(\xi, t) \tilde{\eta}(\xi, x)}{\partial \xi} \eta^{2}(\xi, t)+ \\
\frac{\partial^{3} k_{D}(\xi, t) \tilde{\eta}(\xi, x)}{\partial \xi^{3}} \eta(\xi, t)- \\
\frac{\partial^{2} \kappa(\xi, t) \tilde{\eta}(\xi, x)}{\partial \xi^{2}} \eta(\xi, t)
\end{array}\right] d \xi
\end{array}\right] d \xi
$$

with

$$
k_{N}=\frac{3 c_{0}}{2 h} \text { and } k_{D}=\frac{c_{0} h^{2}}{6}
$$

Fig. 2 shows the interaction of two solitons calculated by 1-Step GIRM. Both solitons move to the right and the speed of the bigger one is faster than the speed of the small one (see Eq. (6)). The calculation was conducted in a frame moving with mean speed of the individual solitons. The accuracy of the numerical results obtained by 1 -step GIRM is very high. Initial condition is given by

$$
\eta(x, 0)=\eta_{1} \operatorname{sech}^{2}\left(\sqrt{\frac{3 \eta_{1}}{4 h^{3}}}\left(x-\frac{3 L}{8}\right)\right)+\eta_{2} \operatorname{sech}^{2}\left(\sqrt{\frac{3 \eta_{2}}{4 h^{3}}}\left(x-\frac{5 L}{8}\right)\right)
$$

and values of the parameters are

$$
\begin{aligned}
& g=9.8, \eta_{1}=0.5, \eta_{2}=0.125, h=1, L=64, N=120, d x=L / N, \\
& d t=0.004, \gamma=0.75 d x, T=20000 d t, c_{0}=3.130495, c_{1}=3.913119 \text {, } \\
& c_{2}=3.326151, U=3.619635 \text {. }
\end{aligned}
$$

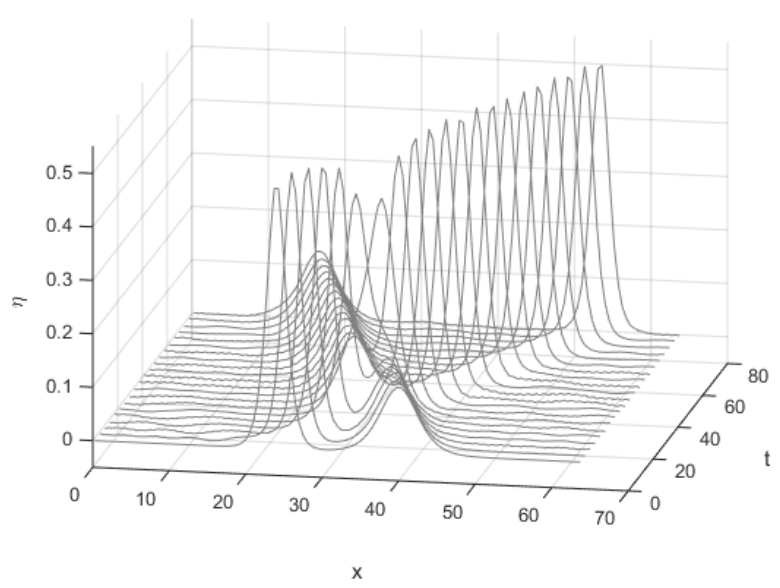

Figure 2. Solution plot of interaction of big and small solitons (by 1-step GIRM).

\subsection{Soliton-to-Bottom Interaction}

We consider an interaction of a soliton to the bottom with three different geometries: bottom geometry 1 . 


$$
h=\left\{\begin{array}{l}
h_{0} \quad \text { if } x<L / 3 \\
h_{0}-\left(0.2 h_{0} /(L / 2-L / 3)\right)(x-L / 3) \quad \text { if } x \leq L / 3<x \leq L / 2 \\
0.8 h_{0} \quad \text { otherwise }
\end{array}\right.
$$

bottom geometry 2

$$
h=\left\{\begin{array}{l}
h_{0} \text { if } x<L / 3 \\
h_{0}+\left(0.2 h_{0} /(L / 2-L / 3)\right)(x-L / 3) \quad \text { if } x \leq L / 3<x \leq L / 2 \\
1.2 h_{0} \quad \text { otherwise }
\end{array}\right.
$$

bottom geometry 3

$$
h=\left\{\begin{array}{l}
h_{0} \quad \text { if } x<L / 3 \\
h_{0}-\left(0.5 h_{0} /(L / 2-L / 3)\right)(x-L / 3) \quad \text { if } x \leq L / 3<x \leq L / 2 \\
0.5 h_{0} \quad \text { otherwise }
\end{array}\right.
$$

and initial condition is given by

$$
\eta(x, 0)=\eta_{0} \operatorname{sech}^{2}\left(\sqrt{\frac{3 \eta_{0}}{4 h^{3}}}\left(x-\frac{L}{6}\right)\right)
$$

Values of parameters used in the experiments are

$$
\begin{array}{r}
g=9.8, \eta_{0}=0.25, \quad h_{0}=1, L=128, N=160, d x=L / N, d t=0.004, \\
T=6000 d t, \quad \gamma=0.75 d x
\end{array}
$$

for bottom geometries 1 and 2 and

$$
\begin{array}{r}
g=9.8, \eta_{0}=0.1, \quad h_{0}=1, L=60, N=80, d x=L / N, d t=0.004, \\
T=3000 d t, \quad \gamma=0.75 d x \quad(27 \mathrm{~b})
\end{array}
$$

for bottom geometry 3 , respectively.

Interactions of a single soliton to the bottom with different geometries are shown in Fig. 3. The tendency of the numerical results by 1 -step GIRM seems reasonable.

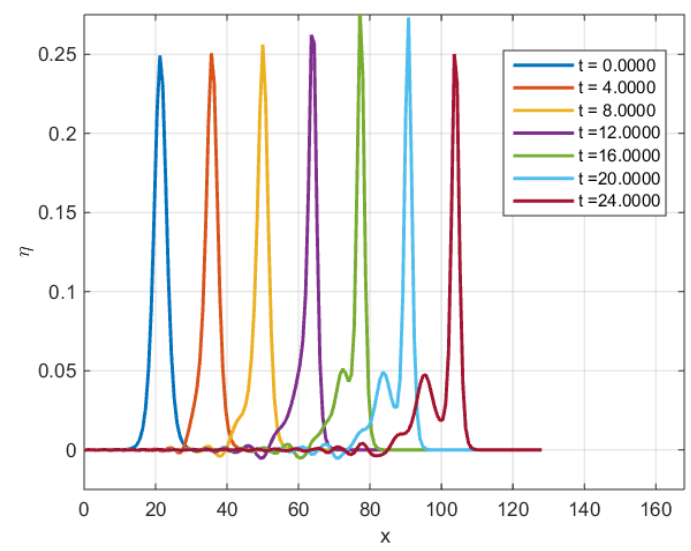

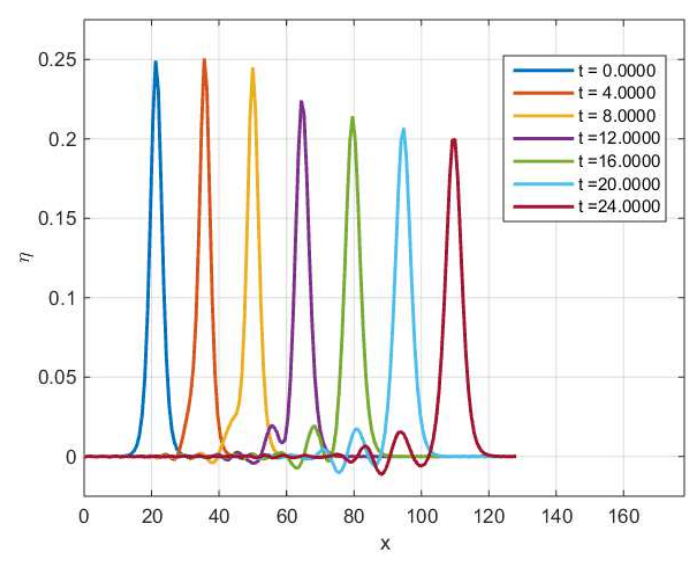

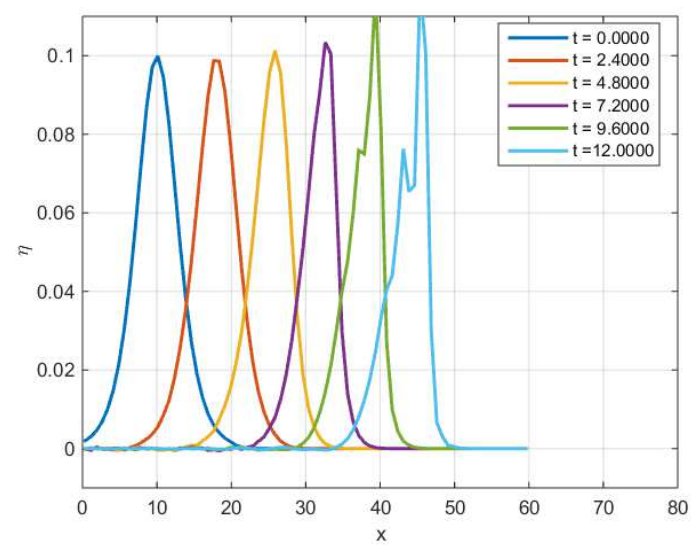

Figure 3. Solution plots of interaction of soliton-to-bottom with different bottom geometries: bottom geometry 1, 2, and 3 (from top to bottom).

\section{Conclusion}

We developed an accurate numerical scheme based on GIRM for solitary wave phenomena including a single soliton propagation, soliton-to-bottom interaction and soliton-to-soliton interaction. In case of a single soliton, the bottom is considered constant in order to understand the behavior of the soliton propagation as it travels in the middle of the sea. In case of soliton-to-bottom interaction, we study behavior of a single soliton propagation when the bottom has different geometries. Finally, we evaluate interaction of two different i.e., big and small solitons. In order to verify the theory, numerical experiments are conducted and accurate approximate solutions are obtained in admissible time in each case of the soliton interactions.

\section{Appendix A. Matlab Code for Propagation of a Single Soliton}

function single_soliton_GIRM ( )

$\%$ Solves single soliton propagation by using 1-step GIRM.

$\mathrm{N}=80$;

eta $0=0.25$;

$\mathrm{dt}=0.004$

$\mathrm{T}=2000$;

$\mathrm{L}=64.0$;
$\%$ number of mesh points

$\%$ initial wave

$\%$ time step

$\%$ total time

$\%$ length of region 


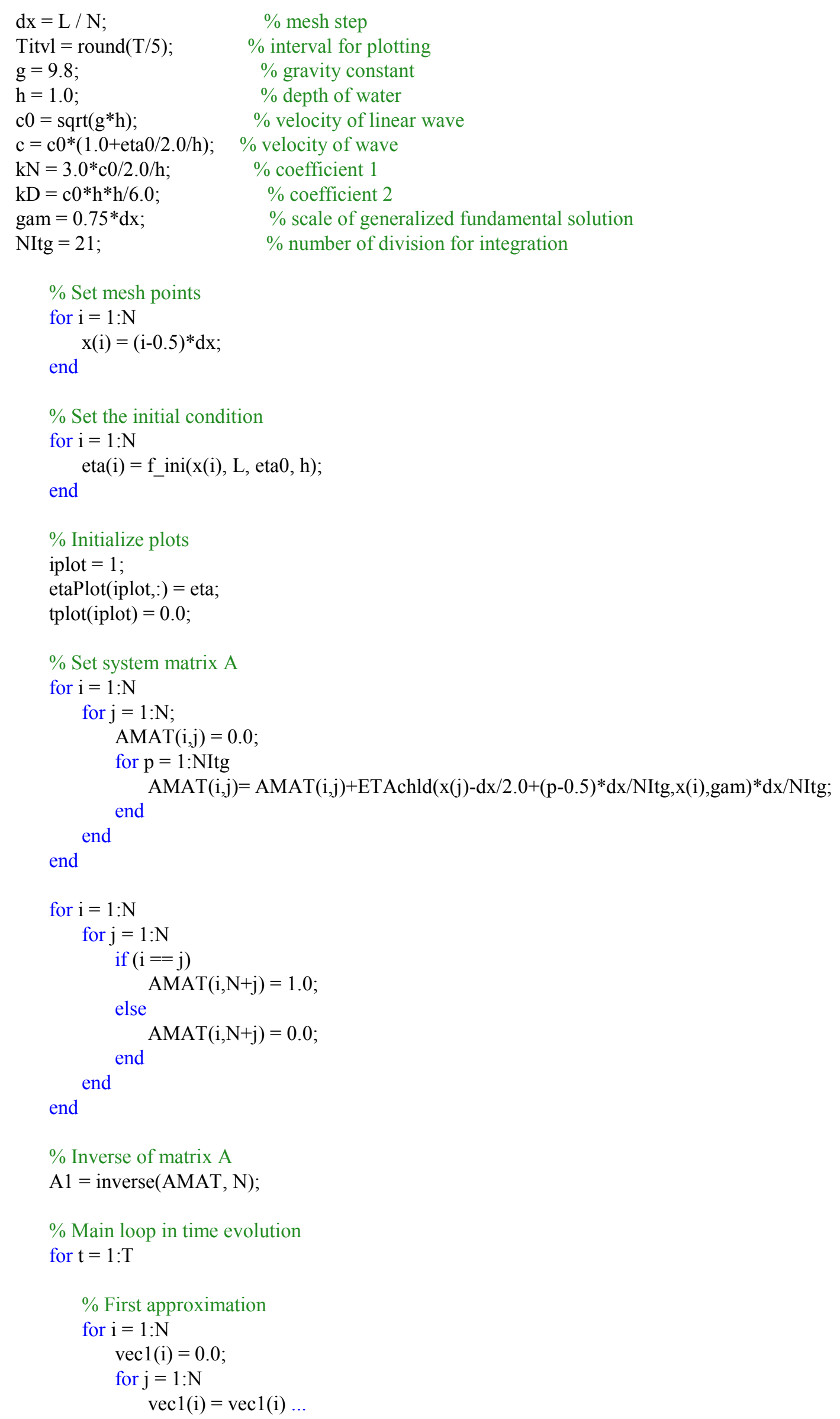




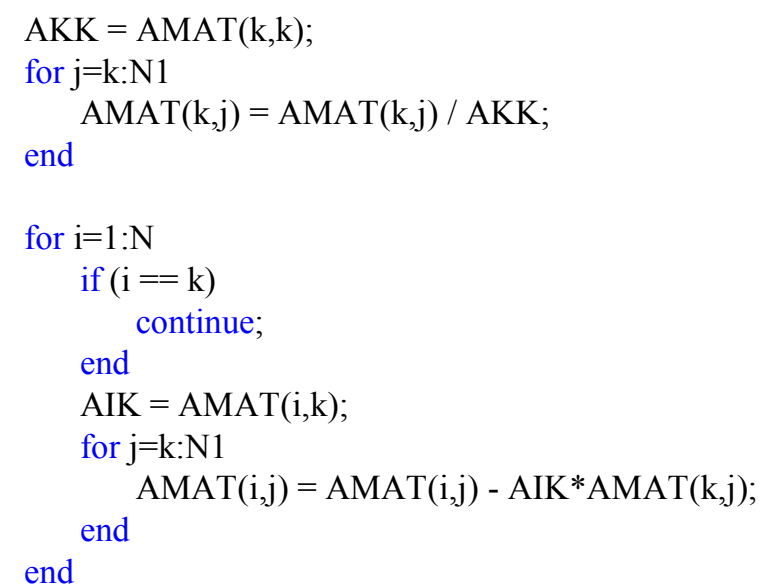

end

$\mathrm{A} 1=\mathrm{AMAT}$

end

function $\mathrm{G}=$ ETAchld( $\mathrm{x}$, xsi, gam)

$\%$ Generalized fundamental solution (GFS)

$\mathrm{G}=1 . / \mathrm{sqrt}\left(2 .{ }^{*} \mathrm{pi}\right) / \mathrm{gam} * \exp (-(\mathrm{x}-\mathrm{xsi}) *(\mathrm{x}-\mathrm{xsi}) / 2 . / \mathrm{gam} / \mathrm{gam})$; end

function $\mathrm{Gx}=$ ETAchld_x( $\mathrm{x}, \mathrm{xsi}$, gam $)$

$\%$ Set 1 st derivative of GFS in $x$

$\mathrm{Gx}=-(\mathrm{x}-\mathrm{xsi}) / \operatorname{sqrt}\left(2 .{ }^{*} \mathrm{pi}\right) /\left(\mathrm{gam}^{\wedge} 3\right) * \exp (-(\mathrm{x}-\mathrm{xsi}) *(\mathrm{x}-\mathrm{xsi}) / 2 . / \mathrm{gam} / \mathrm{gam})$;

end

function $\mathrm{Gxx}=$ ETAchld_xx( $\mathrm{x}, \mathrm{xsi}$, gam $)$

$\%$ Set 2 nd derivative of GFS in $x$

Gxx $=-1 . / \operatorname{sqrt}(2 . * \mathrm{pi}) /\left(\mathrm{gam}^{\wedge} 3\right) * \exp (-(\mathrm{x}-\mathrm{xsi}) *(\mathrm{x}-\mathrm{xsi}) / 2 . / \mathrm{gam} / \mathrm{gam}) \ldots$

$+(\mathrm{x}-\mathrm{xsi}) *(\mathrm{x}-\mathrm{xsi}) / \operatorname{sqrt}\left(2 .{ }^{*} \mathrm{pi}\right) /\left(\mathrm{gam}^{\wedge} 5\right)^{*} \exp (-(\mathrm{x}-\mathrm{xsi}) *(\mathrm{x}-\mathrm{xsi}) / 2 . / \mathrm{gam} / \mathrm{gam})$; end

function Gxxx $=$ ETAchld_xxx( $x$, xsi, gam $)$

$\%$ Set 3 rd derivative of GFS in $x$

$\operatorname{Gxxx}=3 . *(\mathrm{x}-\mathrm{xsi}) / \mathrm{sqrt}\left(2 .{ }^{*} \mathrm{pi}\right) /\left(\mathrm{gam}^{\wedge} 5\right) * \exp (-(\mathrm{x}-\mathrm{xsi}) *(\mathrm{x}-\mathrm{xsi}) / 2 . / \mathrm{gam} / \mathrm{gam}) \ldots$ end

- $(\mathrm{x}-\mathrm{xsi}) *(\mathrm{x}-\mathrm{xsi}) *(\mathrm{x}-\mathrm{xsi}) / \operatorname{sqrt}\left(2{ }^{*}{ }^{\mathrm{p} i}\right) /\left(\mathrm{gam}^{\wedge} 7\right)^{*} \exp (-(\mathrm{x}-\mathrm{xsi}) *(\mathrm{x}-\mathrm{xsi}) / 2 . / \mathrm{gam} / \mathrm{gam})$;

function $\mathrm{f}=\mathrm{f} \operatorname{ini}(\mathrm{x}, \mathrm{L}$, eta $0, \mathrm{~h})$

$\%$ Set initial condition

$\mathrm{f}=\operatorname{eta} 0 * \operatorname{sech}(\operatorname{sqrt}(3 . * \operatorname{eta} 0 / 4 . / \mathrm{h} / \mathrm{h} / \mathrm{h} *(\mathrm{x}-\mathrm{L} / 4)). * \operatorname{sech}(\operatorname{sqrt}(3 . * \operatorname{eta} 0 / 4 . / \mathrm{h} / \mathrm{h} / \mathrm{h} *(\mathrm{x}-\mathrm{L} / 4)) ;$. end

\section{References}

[1] Russell J. S., "Report on Waves." Report of the 14th Meeting of the British Association for the Advancement of Science. London: John Murray, pp. 311-390, 1844.

[2] Korteweg, D. J.; de Vries, G., "On the change of form of long waves advancing in a rectangular canal, and on a new type of long stationary waves." Philosophical Magazine. 39: 422-443, 1895

[3] Demiray H., "Weakly nonlinear waves in water of variable depth: Variable-coefficient Korteweg-de Vries equation." Comput. Math. Appl. 60, 1747-1755, 2010.

[4] Drazin P.G., Johnson R.S., "Solitons: An Introduction." 2nd ed. Cambridge University Press, 1989

[5] Demiray H., "A note on the wave propagation in water of variable depth." Applied Mathematics and Computation 218, 2294-2299, 2011. 
[6] H. Isshiki, "From Integral Representation Method (IRM) to Generalized Integral Representation Method (GIRM)," Applied and Computational Mathematics, Special Issue: Integral Representation Method and Its Generalization, Vol. 4, No. 3-1, 2015, pp. 1-14. doi: 10.11648/j.acm.s.2015040301. 11

[7] H. Isshiki, "Effects of Generalized Fundamental Solution (GFS) on Generalized Integral Representation Method (GIRM)," Applied and Computational Mathematics, Special Issue: Integral Representation Method and Its Generalization. Vol. 4, No. 3-1, 2015, pp. 40-51. doi: 10.11648/j.acm.s.2015 040301.13

[8] H. Isshiki, T. Takiya, and H. Niizato, "Application of Generalized Integral representation (GIRM) Method to Fluid Dynamic Motion of Gas or Particles in Cosmic Space Driven by Gravitational Force," Applied and Computational
Mathematics, Special Issue: Integral Representation Method and Its Generalization. Vol. 4, No. 3-1, 2015, pp. 15-39. doi: 10.11648/j.acm.s.2015040301.12

[9] H. Isshiki, "Application of the Generalized Integral Representation Method (GIRM) to Tidal Wave Propagation," Applied and Computational Mathematics, Special Issue: Integral Representation Method and Its Generalization. Vol. 4, No. 3-1, 2015, pp. 52-58. doi: 10.11648/j.acm.s.20150403 01.14

[10] H. Niizato, G. Tsedendorj, H. Isshiki. Implementation of One and Two-step Generalized Integral Representation Methods (GIRMs). Applied and Computational Mathematics, Special Issue: Integral Representation Method and its Generalization. Vol. 4, No. 3-1, 2015, pp. 59-77. doi: 10.11648/j.acm.s.2015 040301.1 